\title{
Comparative Study of Visual Outcomes Following Vitrectomy for Nucleus Drop Caused by Various Aetiologies
}

\author{
Mehul Shah*, Shreya Shah, Ruhi Gunay and Raunaq Khanna \\ Drashti Netralaya, Dahod, Gujarat, India \\ *Corresponding Author: Mehul Shah, Professor, Drashti Netralaya, Dahod, \\ Gujarat, India. \\ DOI: $10.31080 /$ ASOP.2020.03.0153
}

Received: July 12, 2020

Published: July 28, 2020

(C) All rights are reserved by Mehul Shah., et al.

\begin{abstract}
Purpose: Nucleus drop is a rare and serious complication. We investigated visual outcomes following corrective surgery and compared outcomes between surgeries performed in various aetiologies.

Method: A retrospective cohort study was performed after obtaining the approval of the ethical committee. The data of patients who underwent vitrectomy for nucleus drop were collected from electronic medical records. Patients were grouped based on the following aetiologies: traumatic, iatrogenic, spontaneous, and congenital. After pars plana vitrectomy and nucleus removal, comorbidities were managed either medically or surgically. All data were collected according to a pretested format and exported to SPSS 22 .

Results: Our cohort consisted of 169 eyes of 169 patients. The mean age of patients was $55.36 \pm 15.7$ years. Of 169 patients, 107 (63.3\%) were men and 62 (33.7\%) were women. Of 169 patients, 44 (26\%) were classified as having traumatic nucleus drop and 125 (74\%) developed spontaneous nucleus drop without trauma following cataract surgeries (Table 2). In the iatrogenic group, 33 (38.56\%) patients developed nucleus drop during phacoemulsification and 59 (62.9\%) following MSICS.

Conclusion: The visual outcome following corrective vitrectomy for nucleus drop may be satisfactory if managed well; no significant difference in the outcome was noted based on the aetiology.

Keywords: Nucleus Drop; Traumatic Nucleus Drop; Iatrogenic Nucleus Drop; Visual Outcome
\end{abstract}

\section{Introduction}

Cataracts are responsible for $47 \%$ of all cases of blindness worldwide. The epidemiological effect of cataracts varies among different countries, and its rate of occurrence is associated with economic conditions. All stakeholders aim to improve the quality and quantity of cataract surgery $[1,2]$.

Various postgraduate training programs in cataract surgery are currently available in many countries at different government and nongovernmental institutions, with a wide range of facilities $[1,2]$.

Differences in infrastructure, patient load, and faculty's skill at various training facilities lead to the development of different skill levels and thus varying surgical results. Modern cataract surgery has a steep learning curve.

Monitoring the outcomes of surgical treatment for cataracts is crucial [3]. Although cataract surgery is usually performed by phacoemulsification, manual small-incision cataract surgery
(MSICS) is an important technique often used in developing countries, with its surgical outcome, safety, and efficacy being comparable to those of phacoemulsification $[4,5]$.

Nucleus drop is a serious complication of cataract surgery and may result in severe sight loss. Visual outcomes are poor following the development of nucleus drop [6,7].

Trauma is a cause of monocular blindness in developed countries; however, few studies have addressed the problem of trauma in rural areas [8-11].

Ocular trauma can cause ectopia lentis [8]. Methods used to evaluate visual outcomes in eyes managed for traumatic cases may not be similar [11]; however, damage to other ocular tissues may compromise visual gain in eyes operated on for traumatic cases. Hence, the success rate may differ between eyes with different aetiologies. Traumatic cataract is a crucial cause of vision loss following ocular trauma [11-13]. 
With the introduction of the Birmingham Eye Trauma Terminology System (BETTS), the documentation of ocular trauma has been standardised [5]. Therefore, studying visual outcomes following traumatic nucleus drop by using the BETTS would be valuable. Nucleus drop may result from both open and closed globe injuries $[14,15]$.

\section{Methods}

This retrospective cohort study was approved by the ethical committee of our hospital. We enrolled all patients who developed nucleus drop with any aetiology between 2008 and 2019 Iatrogenic cases following cataract surgery by phacoemulsification or MSICS. We retrieved patients' data from electronic medical records All surgeries performed by two senior surgeons in department of single institution. All data were exported in an Excel sheet and processed using Statistical Package SS 22 (IBM inc). We excluded patients with a follow-up period of less than four weeks following surgical treatment.

All enrolled patients underwent vision examination and an anterior examination using a slit lamp, as well as had their posterior segment findings documented using indirect ophthalmology. When the fundus was not visible, B-scan ultrasonography was used to access the posterior segment. The nucleus was removed through the anterior or posterior route depending on clinical conditions. Pars plana vitrectomies were performed using a noncontact viewing system. We removed the nuclei of varying toughness through anterior or pars plana route using phacofragmentation. We followed up patients following a standard format and schedule.

All comorbidities following ocular trauma or surgical procedures were managed medically or surgically. All cases of nucleus drop resulting from ocular trauma were treated using systemic corticosteroids.

All data were entered in an online pretested form, exported to an Excel spreadsheet, and analysed using SPSS 22. Frequency distribution and cross-tabulation were analysed, and 95\% confidence intervals were calculated. Effects were considered significant if the $P$ value was $<0.05$.

\section{Results}

Our cohort consisted of 169 eyes of 169 patients. The mean age of patients was $55.36 \pm 15.7$ years. Of 169 patients, 107 (63.3\%) were men and $62(33.7 \%)$ were women (Table 1). Of 169 patients, $44(26 \%)$ were classified as having nucleus drop due to trauma and 125 (74\%) developed spontaneous nucleus drop without trauma following cataract surgery (Table 2). In the iatrogenic group, 33

\begin{tabular}{|c|c|c|c|}
\hline \multirow{2}{*}{} & \multicolumn{2}{|c|}{ Sex } & \multirow{2}{*}{ Total } \\
\cline { 2 - 3 } & $\mathbf{F}$ & $\mathbf{M}$ & \\
\hline 00 & 1 & 3 & 4 \\
\hline $0-10$ & 3 & 2 & 5 \\
\hline $21-30$ & 2 & 9 & 11 \\
\hline $31-40$ & 3 & 9 & 12 \\
\hline $41-50$ & 12 & 12 & 24 \\
\hline $51-60$ & 25 & 26 & 51 \\
\hline $61-70$ & 12 & 36 & 48 \\
\hline $71-80$ & 4 & 9 & 13 \\
\hline$>80$ & 0 & 1 & 1 \\
\hline Total & 62 & 107 & 169 \\
\hline
\end{tabular}

Table 1: Age and sex distribution.

\begin{tabular}{|c|c|c|c|}
\hline \multirow{2}{*}{$\begin{array}{c}\text { Post-operative } \\
\text { vision }\end{array}$} & \multicolumn{2}{|c|}{ IOL status } & \multirow{2}{*}{ Total } \\
\cline { 2 - 3 } & Aphakia & Pseudophakia & \\
\hline$<1 / 60$ & 23 & 22 & 45 \\
\hline $1 / 60-3 / 60$ & 10 & 20 & 30 \\
\hline $6 / 60-6 / 36$ & 7 & 15 & 22 \\
\hline $6 / 24-6 / 18$ & 6 & 26 & 32 \\
\hline $6 / 12-6 / 9$ & 5 & 31 & 36 \\
\hline $6 / 6-6 / 5$ & 0 & 2 & 2 \\
\hline LF & 2 & 0 & 2 \\
\hline Total & 53 & 116 & 169 \\
\hline
\end{tabular}

Table 2: Comparative study of visual outcome with insertion of intra ocular lens.

P-0.002.

(38.56\%) and 59 (62.9\%) patients developed nucleus drop during phacoemulsification and following MSICS, respectively. The mean follow-up period was 142 days.

Nucleus drop had varying aetiologies: iatrogenic, spontaneous, congenital, and traumatic in 89 (52.7\%), 27 (16\%), 9 (5.3\%), and $44(26 \%)$ patients, respectively. Nucleus drop was bilateral in 10 (5.9\%) patients.

Between 2008 and 2019, we performed 100946 cataract surgeries. Overall surgical complications amounted to 2998 (2.49\%), of which $89(0.08 \%)$ had nucleus drop; of a total of 12687 trauma cases, $128(1 \%)$ had nucleus drop.

In the entire cohort, we could insert a posterior chamber intraocular lens in 115 (68\%) patients; the remaining 53 (32\%) patients remained aphakic. Of a total of 115 (68\%) eyes in which we inserted a posterior chamber lens, 68 (40.2\%) were scleral-fixated 
lens and 43 (25.4\%) were sulcus-fixated lens. When we compared the visual outcome between aphakic and pseudophakic eyes, the difference was significant $(\mathrm{P}=0.04)$.

When we examined the visual outcomes of all patients following the surgical management of nucleus drop, we found significant improvements $(\mathrm{P}=0.005$, Table 3$)$. We found that 70 (41.4\%) patients improved by more than 6/24, and 45 (26.5\%) did not improve by more than $1 / 60$ because of comorbidities. When we compared the visual outcome with aetiologies, we did not find a significant difference $(P=0.206)$.

We compared visual outcomes by aetiologies between traumatic and nontraumatic causes following surgical management

\begin{tabular}{|l|c|c|c|c|c|c|}
\hline \multirow{2}{*}{ Post operative vision } & \multicolumn{5}{|c|}{ Pre operative vision } & \multirow{2}{*}{ Total } \\
\cline { 2 - 7 } & $\mathbf{<} \mathbf{1 / 6 0}$ & $\mathbf{1 / 6 0}-\mathbf{3 / 6 0}$ & $\mathbf{6 / 6 0}-\mathbf{6 / 3 6}$ & $\mathbf{6 / 2 4} \mathbf{- 6 / 1 8}$ & $\mathbf{6 / 1 2} \mathbf{- 6 / 9}$ & \\
\hline$<1 / 60$ & 26 & 4 & 9 & 6 & 0 & 45 \\
\hline $1 / 60-3 / 60$ & 15 & 5 & 4 & 4 & 2 & 30 \\
\hline $6 / 60-6 / 36$ & 6 & 6 & 8 & 1 & 1 & 22 \\
\hline $6 / 24-6 / 18$ & 8 & 4 & 15 & 5 & 0 & 32 \\
\hline $6 / 12-6 / 9$ & 8 & 11 & 9 & 8 & 0 & 36 \\
\hline $6 / 6-6 / 5$ & 1 & 0 & 1 & 0 & 0 & 2 \\
\hline LF & 1 & 1 & 0 & 0 & 0 & 2 \\
\hline Total & 65 & 31 & 46 & 24 & 3 & 169 \\
\hline
\end{tabular}

Table 3: Comparative study of visual outcome pre and post operative vision.

$$
\mathrm{P}=0.03 \text {. }
$$

non traumatic category and did not find any significant difference between these groups $(P=0.237$, Table 4$)$.

When we compared the visual outcomes of surgical management among different time lags after the primary procedure or trauma, we found no significant difference $(\mathrm{P}=0.135)$.

In nontraumatic cases, no significant difference in the visual outcome following surgical management was observed between primary surgery performed by phacoemulsification or MSICS. (P =
0.122) Difference is insignificant when scleral fixated or other intra ocular lens ( $\mathrm{p}=0.131$, Table 5).

The main causes of non-improvement were corneal edema (18.9\%), secondary glaucoma, and optic atrophy (9.46\%).

\section{Discussion}

In this patient cohort, we determined that crystalline lens dislocation occurred due to various reasons such as ocular trauma, iatrogenic complications during surgery, and spontaneous or con-

\begin{tabular}{|c|c|c|c|c|c|c|}
\hline \multirow{2}{*}{ Post operative vision } & \multicolumn{5}{|c|}{ Aetiology } & \multirow{2}{*}{ Total } \\
\hline & Congenital & Iaterogenic & Spontaneous & Spontaneus & Traumatic & \\
\hline$<1 / 60$ & 3 & 20 & 0 & 6 & 16 & 45 \\
\hline $1 / 60-3 / 60$ & 2 & 17 & 0 & 5 & 6 & 30 \\
\hline $6 / 60-6 / 36$ & 0 & 11 & 1 & 5 & 5 & 22 \\
\hline $6 / 24-6 / 18$ & 0 & 20 & 0 & 4 & 8 & 32 \\
\hline $6 / 12-6 / 9$ & 3 & 19 & 0 & 6 & 8 & 36 \\
\hline $6 / 6-6 / 5$ & 0 & 1 & 0 & 0 & 1 & 2 \\
\hline $\mathrm{LF}$ & 0 & 1 & 0 & 1 & 0 & 2 \\
\hline Total & 8 & 89 & 1 & 27 & 44 & 169 \\
\hline
\end{tabular}

Table 4: Comparative study of visual outcome according to aetiology.

$$
\mathrm{P}=0.783 \text {. }
$$




\begin{tabular}{|c|c|c|c|}
\hline \multirow{2}{*}{ Post operative vision } & \multicolumn{2}{|c|}{ Scleral fixation } & \multirow{2}{*}{ Total } \\
\cline { 2 - 3 } & SF IOL & ELSE & \\
\hline$<1 / 60$ & 13 & 32 & 45 \\
\hline $1 / 60-3 / 60$ & 14 & 16 & 30 \\
\hline $6 / 60-6 / 36$ & 8 & 14 & 22 \\
\hline $6 / 24-6 / 18$ & 11 & 21 & 32 \\
\hline $6 / 12-6 / 9$ & 21 & 15 & 36 \\
\hline $6 / 6-6 / 5$ & 1 & 1 & 2 \\
\hline LF & 0 & 2 & 2 \\
\hline Total & 68 & 101 & 169 \\
\hline
\end{tabular}

Table 5: Comparative study of visual outcome with scleral fixated intra ocular lens.

$$
\mathrm{P}=0.131 \text {. }
$$

genital causes. To our knowledge, no study has reported all aetiologies and compared visual outcomes among different aetiologies.

All other studies have included less number of cases.

Various authors have reported lens dislocation because of following reasons.

In our study, nucleus drop occurred in 128 (1\%) patients due to ocular trauma and as a surgical complication in $89(0.08 \%)$ patients. Rotim reported that $0.68 \%$ of patients who underwent MSICS had lens dislocation, and Hashemi reported that the incidence of lens dislocation was $3.72 \%$ in an Iranian cataract surgery survey $[18,19]$.

Both Rotim and Hashemi have reported that nucleus posterior dislocation occurred during MSICS [18,19]. Furthermore, Fesharak and Hashemi have reported that nucleus fragment dislocation occurred during phacoemulsification $[19,20]$.

Nucleus drop per operatively has been reported by many authors in case of posterior polar cataract. In addition, various measures to prevent and methods for managing nucleus dislocation have been described by various authors. Malhotra., et al. reported the occurrence of nucleus drop in posterior polar-type cataracts; however, we found that nucleus drop is associated with all types of morphology because many surgeries are performed by junior surgeons $[7,21-24]$.

Kagmeni and Rosignoli have reported that lens dislocation following ocular trauma occurred due to various objects such as a boxer and an exercise belt $[25,26]$.
Shanmugam reported the occurrence of traumatic dislocation in the suprachoroidal space, and Bawankar reported that nucleus drop occurred in the subretinal region; however, we did not find any nucleus drop in these locations $[27,28]$.

Dropped fragments may cause complications such as retinal ischaemia, secondary glaucoma, and retinal breaks [29-32].

Ke and Fujikawa have reported nucleus dislocation in the vitreous cavity following ocular trauma or spontaneously [32-34]. Weave predicted injury risk by performing computational simulations of ocular blast loading risk of dislocated lens with model is 1 to $100 \%$ [35].

$\mathrm{Wu}$, Kuruvilla, and Bhattacharjee have reported the occurrence of nucleus dislocation in the anterior segment either because of trauma or spontaneously [36-38].

Radiodiagnosis may be performed through ultrasonography bscan or CT scan with good sensitivity and specificity [39-41].

Pati, Gurunadh, and Kaynak have reported the management of nucleus drop in the vitreous cavity by using the pars plana route 23 gauge hand piece and anteriorly by using anterior vitrectomy techniques such as those employed in the current study [42-44].

Specific histopathological and chemical factors may be responsible for weak zonules [45].

Visual outcomes significantly improved following surgical management $[6,7]$.

The visual outcome was not significantly different among different aetiologies $(\mathrm{P}=0.606)$. We are not aware of any other study that examined this variable. In cases of ocular trauma, the lens is not the only structure that affects the visual outcome $[16,17]$.

When we examined the time duration between primary insults and corrective surgery, we found no significant effect of a greater time lag $(\mathrm{P}=0.135)$ on later vision. This may be because inflammation following the primary insult had subsided during the early period. When vitrectomy is performed late, visibility is better and no retinal detachment is found, suggestive of a better prognosis. Shah., et al. suggested late intervention in traumatic cases [17].

We did not find significant differences due to vitrectomy gauge, primary procedure, or presence or absence of an intraocular lens. Furthermore, we did not find any other study with which to compare these findings. 
When we compared vision with pseudophakia and best-corrected aphakia, we found a significant difference $(\mathrm{P}=04)$.

We used intra ocular lens in sulcus- and scleral-fixated lens for the management of aphakia but did not find a significant difference with the type of lens used ( $\mathrm{P}=0.237)$.

Other authors used iris claw lens fixated on the posterior surface of the iris or artisan lens for the management of aphakia [46]. Agarwal., et al. also reported the usage of a glued lens and its learning curve [47], and Farrah compared the outcome between the iris claw lens and scleral-fixated lens [48]. Fiorentzis reported lens insertion according to retained anatomical structures, and similar guidelines were used in the current study [49].

The reason for non improvement of vision was comorbidities in traumatic cases and surgical damage to other tissues during primary surgery in iatrogenic cases.

\section{Conclusion}

Although nucleus drop is a serious complication, if it is managed well, a favourable visual outcome can be achieved. No difference was observed in visual outcomes between various aetiologies.

\section{Bibliography}

1. Lawani R., et al. "Magnitude and strategies of cataract management in the world". Medecine Tropicale (Mars) 67 (2007): 644-650.

2. Shah MA., et al. "Final visual outcome following re exploration of cataracts performed by trainee surgeons in India". Indian Journal of Medical Sciences 65 (2011): 365-370.

3. Bulletin of the World Health Organization 77.6 (1999).

4. Hennig A., et al. "Suture less cataract surgery with nucleus extraction: outcome of a prospective study in Nepal". British Journal of Ophthalmology 87 (2003): 266-270.

5. Gogate PM., et al. "Safety and efficacy of phacoemulsification compared with manual small incision cataract surgery by a randomized controlled clinical trial: six-week results". Ophthalmology 112 (2005): 869-874.

6. Kalantan H. "Posterior polar cataract: A review". Saudi Journal of Ophthalmology 26.1 (2012): 41-49.
7. Das S., et al. "Surgical and visual outcomes for posterior polar cataract". British Journal of Ophthalmology 92 (2008): 14761478.

8. Khatry SK., et al. "The epidemiology of ocular trauma in rural Nepal”. British Journal of Ophthalmology 88 (2004): 456-460.

9. Abraham DI., et al. "Epidemiology of eye injuries in rural Tanzania”. Ophthalmic Epidemiology 6 (1999): 85-94.

10. D Virgil Alfaro., et al. "Fishing-related ocular trauma". American Journal of Ophthalmology 139 (2005): 488-492.

11. Shah M., et al. "Ocular injuries and visual status before and after Their management in the tribal areas of Western India-A historical cohort study". Graefe's Archive for Clinical and Experimental Ophthalmology 246 (2008): 191-197.

12. Kuhn F., et al. "The Birmingham Eye Trauma Terminology system (BETT)”. Journal Français D'Ophtalmologie 27 (2004): 206-210.

13. Shah M., et al. "Visual recovery and predictors of visual prognosis after managing traumatic cataracts in 555 patients". Indian Journal of Ophthalmology 59 (2011): 217-222.

14. Shah MA., et al. "Ocular Trauma Score as a predictor of final visual outcomes in traumatic cataract cases in pediatric patients". Journal of Cataract and Refractive Surgery 38 (2012): 959-965.

15. Shah MA., et al. "Ocular Trauma Score: a useful predictor of visual outcome at six weeks in patients with traumatic cataract". Ophthalmology 119 (2012): 1336-1341.

16. Shah MA., et al. "Comparative study of final visual outcome between open- and closed-globe injuries following surgical treatment of traumatic cataract". Graefe's Archive for Clinical and Experimental Ophthalmology 249 (2011): 1775-1781.

17. Shah MA., et al. "Effect of interval between time of injury and timing of intervention on final visual outcome in cases of traumatic cataract". European Journal of Ophthalmology 21 (2011): 760-765.

18. Rotimi-Samuel A., et al. "Nucleus drop during small incision cataract surgery: A report of four cases". Nigerian Postgraduate Medical Journal 22.4 (2015): 237-240. 
19. Hashem H., et al. "Intraoperative Complications of Cataract Surgery in Tehran Province, Iran". Optometry and Vision Science 93 (3): 266-271.

20. Fesharaki H., et al. "A comparative study of complications of cataract surgery with phacoemulsification in eyes with high and normal axial length". Advanced Biomedical Research 1 (2012): 67.

21. Malhotra C., et al. "Phacoemulsification in posterior polar cataract: Experience from a tertiary eye care Centre in North India”. Indian Journal of Ophthalmology 68.4 (2020): 589-594.

22. Sachdev MS., et al. "Femtosecond laser-integrated anterior segment optical coherence tomography to detect preexisting posterior capsular dehiscence and increase safety in posterior polar cataracts". Journal of Cataract and Refractive Surgery 46 (2): 235-240.

23. Das S., et al. "Surgical and visual outcomes for posterior polar cataract". British Journal of Ophthalmology 92.11 (2008): 1476-1478.

24. Kapoor G., et al. "Posterior polar cataract: Minimizing risks". Medical Journal Armed Forces India 72.3 (2016): 242-246.

25. Kagmeni G., et al. "Ophthalmological findings in Cameroonian boxers”. Clinical Ophthalmology 11 (2017): 1121-1126.

26. Rosignoli LM., et al. "Exercise band-induced lens dislocations: A case series". American Journal of Ophthalmology Case Reports 15 (2019): 100496.

27. Shanmugam MP., et al. "Post-traumatic suprachoroidal dislocation of crystalline lens and its management". Indian Journal of Ophthalmology 67.9 (2019): 1469-1470.

28. Bawankar P., et al. "A rare case of traumatic subretinal migration of crystalline lens, corroborated histologically". Indian Journal of Ophthalmology 65.12 (2017): 1495-1497.

29. Arranz-Marquez E., et al. "Late onset lens particle glaucoma in Marfan syndrome”. Archivos de la Sociedad Espanola de Oftalmologia 90.1 (2014): 40-43.

30. Ochi R., et al. "Case of retinal ischemia and retinal arteritis secondary to intravitreal nuclear drop". Nippon Ganka Gakkai Zasshi 115.12 (2011): 1101-1104.
31. Kuiper J and M Slabaugh. "Secondary Angle Closure due to Crystalline Lens Dislocation in a Patient with Atopic Dermatitis and Chronic Eye Rubbing". Case Reports on Ophthalmology 9.1 (2018): 197-201.

32. Ke G., et al. "Retinal break associated with traumatic lens dislocation or subluxation requiring vitrectomy". Graefe's Archive for Clinical and Experimental Ophthalmology 258.3 (2020): 693-697.

33. Fujikawa A., et al. "Visual outcomes and prognostic factors in open-globe injuries". BMC Ophthalmology 18.1 (2018): 138.

34. Choovuthayakorn J., et al. "Epidemiology of Eye Injuries Resulting in Hospitalization, a Referral Hospital-Based Study". Clinical Ophthalmology 14 (2020): 1-6.

35. Weaver AA., et al. "Injury risk prediction from computational simulations of ocular blast loading". Biomechanics and Modeling in Mechanobiology 16.2 (2017): 463-477.

36. Wu S., et al. "Corneal decompensation due to spontaneous absorption of lens and anterior dislocation of lens capsule: A case report". Medicine (Baltimore) 98.50 (2019): e18417.

37. Kuruvilla SE., et al. "Microcornea and bilateral ectopia lentis in an infant: unusual severe ocular presentation of neonatal Marfan syndrome". Journal of AAPOS 23.2 (2019): 107-108.

38. Bhattacharjee H., et al. "Bilateral spontaneous anterior dislocation of intraocular lens with the capsular bag in a patient with pseudoexfoliation". Indian Journal of Ophthalmology 63.10 (2015): 796-798.

39. Ojaghihaghighi S., et al. "Diagnosis of Traumatic Eye Injuries with Point-of-Care Ocular Ultrasonography in the Emergency Department". Annals of Emergency Medicine 74.3 (2019): 365371.

40. Gad K., et al. "CT in the Evaluation of Acute Injuries of the Anterior Eye Segment". American Journal of Roentgenology 209.6 (2017): 1353-1359.

41. Thelen J and AA Bhatt. "Acute ocular traumatic imaging: what the radiologist should know [corrected]". Emergency Radiology 24.5 (2017): 585-592. 
42. Patil KB., et al. "Pars plana vitrectomy with posterior iris claw implantation for posteriorly dislocated nucleus and intraocular lens". Indian Journal of Ophthalmology 59.6 (2011): 497500 .

43. Gurunadh VS., et al. "Management of Nucleus and IOL Drop". Medical Journal Armed Forces India 64.4 (2008): 315-316.

44. Kaynak S., et al. "Staining of vitreous with triamcinolone acetonide in retained lens surgery with phacofragmentation". Journal of Cataract and Refractive Surgery 32.1 (2006): 56-59.

45. Bhattacharjee H., et al. "Pathology and immunohistochemistry of capsular bag in spontaneously late dislocated capsular bagintraocular lens complex". Indian Journal of Ophthalmology 65.10 (2017): 949-954.

46. Kelkar A., et al. "Primary iris claw IOL retrofixation with intravitreal triamcinolone acetonide in cases of inadequate capsular support". International Ophthalmology 38.1 (2018): 111-117.

47. Agarwal A., et al. "Glued intraocular lens scaffolding to create an artificial posterior capsule for nucleus removal in eyes with posterior capsule tear and insufficient iris and sulcus support". Journal of Cataract and Refractive Surgery 39.3 (2013): 326-33.

48. Farrahi F., et al. "Iris Claw versus Scleral Fixation Intraocular Lens Implantation during Pars Plana Vitrectomy". Journal of Ophthalmic and Vision Research 7.2 (2012): 118-124.

49. Fiorentzis M., et al. "Methods of fixation of intraocular lenses according to the anatomical structures in trauma eyes". Clinical Anatomy 31.1 (2017): 6-15.

\section{Assets from publication with us}

- Prompt Acknowledgement after receiving the article

- Thorough Double blinded peer review

- Rapid Publication

- Issue of Publication Certificate

- High visibility of your Published work

Website: www.actascientific.com/

Submit Article: www.actascientific.com/submission.php

Emaill us: editor@actascientific.com

Contact us: +919182824667

Citation: Mehul Shah., et al. "Comparative Study of Visual Outcomes Following Vitrectomy for Nucleus Drop Caused by Various Aetiologies". Acta Scientific Ophthalmology 3.8 (2020): 41-47. 